\title{
Predictors of Severity in COVID-19 patients in Pneumology Department Marrakesh, Morocco
}

\author{
C. Rachid $^{1 *}$, PP. Koumeka ${ }^{1}$, S. Ait batahar ${ }^{1}$, L. Amro ${ }^{1}$
}

${ }^{1}$ Pneumology Department, Arrazi Hospital, Chu Mohammed VI, LRMS, FMPM UCA, Marrakesh Morocco

DOI: $10.36347 /$ sjmcr.2022.v10i01.001

| Received: 29.11.2021 | Accepted: 05.01.2022 | Published: 10.01.2022

*Corresponding author: $\mathrm{C}$. Rachid

Pneumology Department, Arrazi Hospital, Chu Mohammed VI, LRMS, FMPM UCA, Marrakesh Morocco

\section{Abstract}

Background: The SARS-CoV-2 coronavirus, has been identified since 2019, as the causative agent of a new respiratory disease called the COVID-19 (Coronavirus Disease 2019). Numerous risk factors for COVID-19 severity have been described, but data on infected patients in North Africa are limited. We aimed to explore the predictive factors of disease severity in COVID-19 patients in the pneumology department of the Mohammed VI Hospital in Marrakech. Methods: A descriptive and analytical cross-sectional study conducted among patients hospitalized for COVID- 19 during the period from October 2020 to December 2020 in intensive care unit CHU Mohammed VI of Marrakech confirmed and presenting acute respiratory distress. The variables retained in the final model were selected using a stepwise forward method with an entry threshold at 0.2 and an exit threshold at 0.05 . The significance threshold was retained for $p<0,05$. The descriptive analysis consisted of the calculation of absolute and relative frequencies for the qualitative variables, and of the positioning and dispersion parameters for the quantitative variables (mean, standard deviation). For the comparison of percentages in bivariate analysis, Pearson's Chi2 statistical test and Fisher's test if necessary were used. Results: 125 cases of COVID-19 were collected (40.8 women and 59.2 men). The average age was 59.6 years (26-90). 86.4\% were exposed to tobacco. $66.7 \%$ were active smokers and $33.3 \%$ were passive smokers. The presence of comorbidities was noted in $77.6 \%$ of cases. These were arterial hypertension $(28.4 \%)$, diabetes $(32.6 \%)$, heart disease $(7.1 \%)$, neoplasia (5.7\%), dysthyroidism (5.7\%) and bronchial asthma (6.4\%). Confirmatory examinations included suggestive imaging in $76.9 \%, 47.9 \%$ a positive pcr. The predominant respiratory symptoms were cough in $84 \%$ of cases, followed by dyspnea in $70.9 \%$ of cases. The predominant extra-respiratory signs were asthenia in $79 \%$ of cases, followed by myalgia in $46 \%$ of cases, anosmia in $29.4 \%$ of cases and agueusia in $21 \%$ of cases. Fever was found in $68 \%$ of patients. $35 \%$ were tachycardic at $>90$ beats $/ \mathrm{min}$ and $43.2 \%$ of patients had a saturation $<90$ beats/min, $16 \%$ a sa02 between $90-95 \% .71$ patients with saturation $>90 \%$ had a mean age of 57.3 years while 54 patients with sao $2<90 \%$ had a mean age of 62.7 with one ( $\mathrm{p}=0.027)$. 42 patients with sao2 $<90 \%$ had a mean lymphocyte count of 1123 with one $(\mathrm{p}=0.003)$. Chest $\mathrm{CT}$ revealed a ground glass appearance in $83.2 \%$, alveolar condensations in $40 \%$. Lesions were critical in $12.8 \%$, severe in $30.8 \%$, moderate in $30.8 \%$, and mild in $25.6 \%$. Radiological images in favor of pulmonary superinfections were noted in $77 \%$ of cases. Radiological extension correlated with saturation: $76.1 \%$ of patients with sa02 $>90 \%$ had mild to moderate pulmonary extension with a $(\mathrm{p}=0.0001)$. Biological disturbances were also noted. These included elevated D-dimer levels in $(31 \%)$, lymphopenia $(47.4 \%)$, leukopenia (8\%), thrombocytopenia (13\%), PNN hyperleukocytosis (34.2\%), liver cytolysis (10\%), elevated CRP (54\%), elevated fibrinogen (59\%), and impaired renal function (36\%). The evolution was marked by admission to the intensive care unit for all patients with the implementation of ventilatory assistance in $91 \%$ of patients. $84 \%$ of patients had an $\mathrm{O} 2$ flow rate <10 1/min with mild to moderate pulmonary extension with one ( $\mathrm{p}=0.011)$ and an OR of 3,94 . Only one case of death was noted. The factors that conditioned the prognosis were: age, lymphocyte count, d-dimer, respiratory rate, saturation and 02 flow.92 patients with a mean age of 57.9 years $+/-13.03$ had a good prognosis versus 33 patients with a mean age of 64.52 years $+/-13.97$ had a poor prognosis with a $(\mathrm{p}=0.016)$. Lymphocyte count was also a factor that conditioned prognosis: 44 patients with a mean lymphocyte count of 1514 had a good prognosis versus 21 poor prognosis patients with a mean lymphocyte count of 1120 with a $(p=0.037)$. D-dimer assay: 37 patients with a mean d-dimer level of 1265 had a good prognosis versus 18 patients with a mean $\mathrm{D}$-dimer level of 9193 with one ( $\mathrm{p}=0.023)$. 02 saturation: 83.1\% (59) of patients with a saturation $>90 \%$ had a good prognosis compared to $61.1 \%$ (33) of patients with a saturation $<90 \%$ with a ( $\mathrm{p}=0.08$ ). Discharge with an oxygen extractor was considered a poor prognostic element in $71 \%$ of patients with a $(\mathrm{p}=0.0001)$ and an OR of 9. Conclusion : The progression of patients with COVID-19 to worsening appears to be associated with age, cardiovascular and metabolic history, severity of radiological involvement, and observed biological disturbances. This study therefore shows the importance of predictive factors such as age, lymphocyte and d-dimer levels, respiratory rate and oxygen saturation as prognostic factors for the evolution of Covid-19 infection with rapid worsening synonymous with the severity of the evolution.

Keywords: Predictors, Severity, Pneumology.

Copyright $(\odot 2022$ The Author(s): This is an open-access article distributed under the terms of the Creative Commons Attribution 4.0 International License (CC BY-NC 4.0) which permits unrestricted use, distribution, and reproduction in any medium for non-commercial use provided the original author and source are credited.

\section{INTRODUCTION}

Since December 2019, the emergence of a new coronavirus strain called SARS-CoV-2 coronavirus, which is highly pathogenic, has been identified as the causative agent of a new respiratory disease: the COVID-19 (Coronavirus Disease 2019).

First appearing in Wuhan in China's Hubei province, this strain then spread to the rest of the world in the following months. On March 11, 2020, the WHO 
qualified the situation as a true pandemic, and containment was adopted everywhere.

At the time of this work, this health crisis has already claimed more than 3.5 million lives worldwide. This daily death toll is increasing with the appearance of new variants and mutants and despite a promising vaccine campaign, which started at the beginning of the year.

If the symptoms can go from a simple flu-like syndrome made of cough, fever or arthralgias, they can be atypical and translate the attack of all the systems. For example, some patients may present with moderate or severe respiratory involvement, ranging from pneumonia to acute respiratory distress syndrome (ARDS). High flow oxygen therapy or respiratory assistance is then necessary to improve the vital prognosis.

In addition, there is a disturbed biological balance, reflecting an excessive inflammatory response, described as a real cytokine storm. This storm is the consequence of an unbalanced innate and adaptive immune response, responsible for irreversible tissue damage leading to multivisceral failure.

In addition to the clinical picture, biological markers or biomarkers reflect the excessive inflammatory response but also the immune status of the host. They are used to guide therapy but also to predict the severity of COVID-19 pneumonia.

Our study was motivated by the reappearance of COVID-19 positive cases in our country in the period following mid-July 2020. Because this time, and contrary to the beginning of the crisis, more severe and therefore fatal forms were observed.

The development of predictive models for severe forms of COVID19 would be a way to rationalize patient management and to relieve the care facilities receiving these patients, whose capacities, including those in intensive care, are very quickly exceeded during a COVID pandemic.

The present study reports the data of 125 patients with laboratory-confirmed SARS-CoV-2 infection admitted to intensive care.

The objective of our study is to analyze all these clinical, biological and radiological parameters and to determine the most sensitive and specific ones to predict the severity of the COVID-19 disease and those that contributed to the prognostic evolution in the Mohammed VI hospital of Marrakesh.

\section{Materials and Methods}

A retrospective descriptive and analytical cross-sectional study conducted among patients hospitalized for COVID-19 during the period from October 2020 to December 2020 in the intensive care unit of the Marrakech University Hospital with acute respiratory distress.

\section{Inclusion criteria}

Patients with positive sars-cov $2 \mathrm{CRP}$ or suggestive imaging. Statistical analysis was performed using SPSS version 19.0 software.

The descriptive analysis consisted of the calculation of absolute and relative frequencies for the qualitative variables, and of the positioning and dispersion parameters for the quantitative variables (mean, standard deviation).

Thus, the variables whose association was significant at the $20 \%$ threshold in bivariate analysis were included in a multivariate model. The threshold of significance was retained for a $\mathrm{p}<0.05$.

The variables retained in the final model were selected using a stepwise forward method with an entry threshold of 0.2 and an exit threshold of 0.05 .

For the comparison of percentages in bivariate analysis, Pearson's Chi2 statistical test and Fisher's test if necessary were used. This method is used when the prognosis of the patients is to be predicted according to certain values or a set of explanatory variables. It suitable for models in which the variables are dichotomous (good/poor prognosis).

\section{RESULTS}

\section{Demographic and clinical characteristics}

In this study, we reported 125 patients with confirmed SARS-CoV-2 infection. The median age was $59.6+/-13.53$ (26-90) years with a median of 61 years and $75(60 \%)$ were men. $66.7 \%$ were active smokers and $33.3 \%$ were passive smokers . 92 (73.6\%) nonsevere patients were discharged from the ICU after completing treatment, $11(8.8 \%)$ were admitted to single rooms, and $21(16.8 \%)$ were placed in the resuscitation unit, with only one death, due to the development of organ dysfunction.

Of the 125 patients, $61 \quad(77.6 \%)$ had comorbidities, such as hypertension ( $\mathrm{n}=35 ; 28 \%)$, diabetes $(\mathrm{n}=41 ; 32.6 \%)$ and heart disease $(\mathrm{n}=8 ; 7.1 \%)$, neoplasia ( $\mathrm{n}=7 ; 5.7 \%)$, dysthyroidism $(\mathrm{n}=6 ; 5.7 \%)$, bronchial asthma $6.4 \%$. The confirmatory examinations included in $76.9 \%$ an evocative imaging, $47.9 \%$ a positive pcr.

The predominant respiratory symptoms were cough in $84,6 \%$ of cases, followed by dyspnea in $70.9 \%$ of cases. The predominant extra-respiratory signs were asthenia in $79 \%$ of cases, followed by myalgia in $46 \%$ of cases, anosmia in $29.4 \%$ of cases and agueusia in $21 \%$ of cases. Fever was found in $68 \%$ of patients. $35 \%$ 
were tachycardic at $>90$ beats $/ \mathrm{min}$ and $43.2 \%$ of patients had a saturation $<90$ beats/min, $16 \%$ a sa02 between $90-95 \%$.

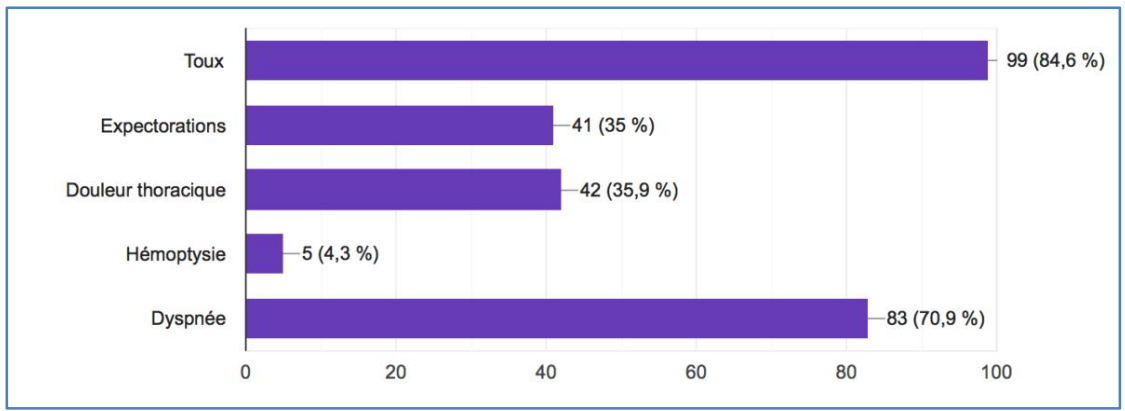

Graph-1: The predominant respiratory symptoms

Regarding the clinical examination parameters, there was a significant difference, 71 patients with a saturation $>90 \%$ had a mean age of 57.3 years while 54 patients with a sao $2<90 \%$ had a mean age of 62.7 with one $(p=0.027)$. This difference was significant.

Table-1: Comparison between age and capillary blood saturation

\begin{tabular}{|l|l|l|l|l|l|}
\hline & Saturation & n & Average & Standard deviation & $\boldsymbol{p}$ \\
\hline \multirow{2}{*}{ AGE } & $<90$ & 54 & 62,7 & 13,2 & \multirow{2}{*}{$\mathbf{0 , 0 2 7}$} \\
\cline { 2 - 6 } & $>=90$ & 71 & 57,3 & 13,4 & \\
\hline
\end{tabular}

\section{Clinical and laboratory findings}

Biological disturbances were also noted. These included elevated D dimers in $(31 \%)$ of cases, lymphopenia (47.4\%), leukopenia (8\%), thrombocytopenia (13\%), PNN hyperleukocytosis (34, $2 \%)$, liver cytolysis (10\%), elevated CRP (54\%), elevated fibrinogen $(59 \%)$, and impaired renal function $(36 \%)$.

With regard to biological parameters, there was a significant difference, 42 patients with a saturation < $90 \%$ had a mean lymphocyte count of 1123 , compared with 59 patients with a saturation >
$90 \%$ with a $(\mathrm{p}=0.003) .24$ patients with saturation < $90 \%$ had a mean platelet count at 234541 , versus 27 patients with saturation $>90 \%$ with a mean platelet count at 320259 with one $(\mathrm{p}=0.032) .24$ patients with saturation $<90 \%$ had a mean NPC count at 8354 with one $(p=0.031)$. The initial crp level also conditioned the prognosis. 48 patients with an initial saturation $<90$ had a mean crp level at 185.5 with one $(\mathrm{p}=0.0001)$. Regarding the initial D-dimer level, 23 patients had an initial saturation $<90$ with a mean of $7209(p=0.037)$.

\section{Correlations between clinical and laboratory} parameters

Table-2: Comparison of capillary blood saturation and biological findings

\begin{tabular}{|c|c|c|c|c|c|}
\hline & Saturation & $\mathbf{n}$ & Average & Standard deviation & $p$ \\
\hline \multirow{2}{*}{ Lymphocytes } & $<90$ & 42 & 1123,6 & 642,8 & \multirow{2}{*}{$\mathbf{0 , 0 0 3}$} \\
\hline & $>=90$ & 59 & 1514,3 & 627,6 & \\
\hline \multirow{2}{*}{ Plates } & $<90$ & 24 & 234541,7 & 109631,0 & \multirow{2}{*}{$\mathbf{0 , 0 3 2}$} \\
\hline & $>=90$ & 27 & 320259,3 & 159338,2 & \\
\hline \multirow{2}{*}{ PNN } & $<90$ & 24 & 8354,7 & 3357,4 & \multirow{2}{*}{$\mathbf{0 , 0 3 1}$} \\
\hline & $>=90$ & 34 & 6451,5 & 3139,4 & \\
\hline \multirow{2}{*}{ Initial CRP } & $<90$ & 48 & 185,5 & 122,6 & \multirow{2}{*}{0,0001} \\
\hline & $>=90$ & 63 & 95,4 & 87,6 & \\
\hline \multirow{2}{*}{ D-Dimer } & $<90$ & 23 & 7209,7 & 12377,8 & \multirow{2}{*}{$\mathbf{0 , 0 3 7}$} \\
\hline & $>=90$ & 32 & 1452,2 & 1372,5 & \\
\hline
\end{tabular}

\section{Clinical and radiological findings}

Chest CT revealed a ground glass appearance in $83.2 \%$, alveolar condensations in $40 \%$. Lesions were critical in $12.8 \%$, severe in $30.8 \%$, moderate in $30.8 \%$, and mild in $25.6 \%$. Radiological images in favor of pulmonary superinfections were noted in $77 \%$ of cases. 


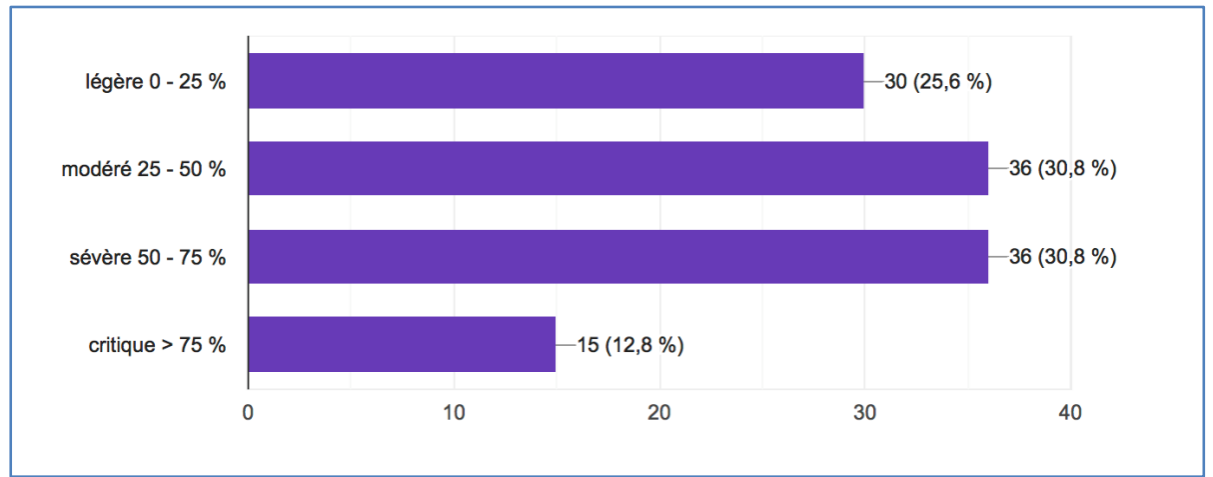

Graph-2: Chest CT extension in COVID 19 disease

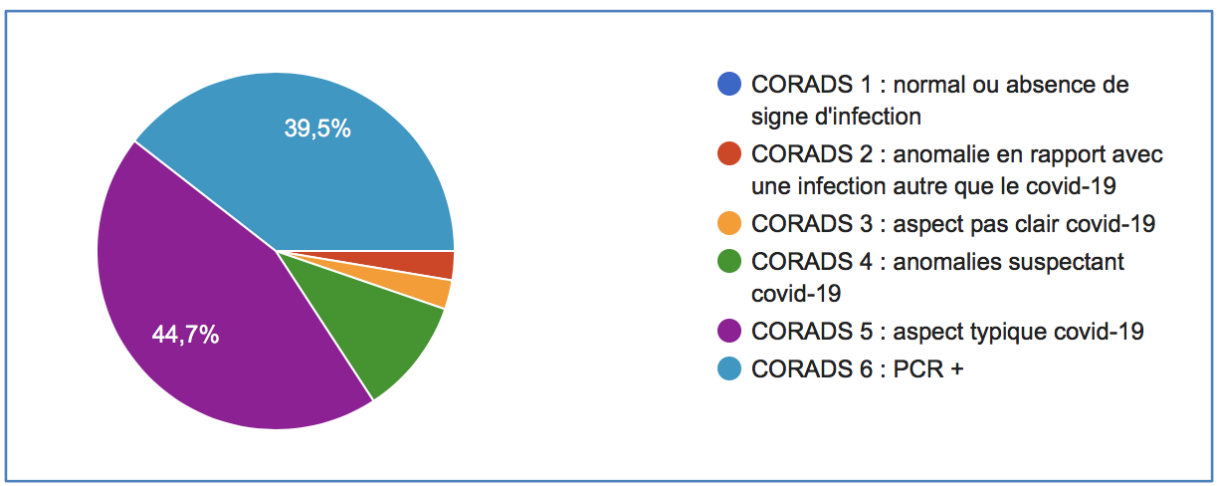

Graph-3: COVID 19 CORADS classification disease

Correlations between clinical and radiological parameters

Radiological extension correlated with saturation: $51(76.1 \%)$ of patients with sa02 > 90\% had mild to moderate pulmonary extension, whereas 38 $(65.5 \%)$ with saturation $<90$ had severe to critical involvement with one $(\mathrm{p}=0.0001)$.

Table-3: Comparison of capillary blood saturation between patients who have mild, severe and critical lung involvement

\begin{tabular}{|c|c|c|c|c|c|}
\hline & \multicolumn{2}{|c|}{ Saturation } & \multirow[t]{2}{*}{$p$} \\
\hline & & & $<90$ & $>=90$ & \\
\hline \multirow{4}{*}{ Pulmonary extension } & mild to & Workforce & 16 & 51 & \multirow{4}{*}{0,0001} \\
\hline & moderate & $\%$ & $23,9 \%$ & $76,1 \%$ & \\
\hline & \multirow{2}{*}{$\begin{array}{l}\text { severe to } \\
\text { critical }\end{array}$} & Workforce & 38 & 20 & \\
\hline & & $\%$ & $65,5 \%$ & $34,5 \%$ & \\
\hline
\end{tabular}

The evolution was marked by admission to the intensive care unit for all patients with the implementation of ventilatory assistance in $91 \%$ of patients. $84 \%$ of patients had an $\mathrm{O} 2$ flow rate $<101 / \mathrm{min}$ with mild to moderate pulmonary extension with one $(\mathrm{p}=0.011)$. Only one case of death was noted.

Table-4: Correlations between 02 flow rate and pulmonary extension

\begin{tabular}{|c|c|c|c|c|c|}
\hline & \multicolumn{2}{|c|}{ O2 flow rate } & \multirow[t]{2}{*}{$p$} \\
\hline & & & $<=10$ & $>10$ & \\
\hline \multirow{4}{*}{$\begin{array}{l}\text { Pulmonary } \\
\text { extension }\end{array}$} & \multirow{2}{*}{ mild to moderate } & Workforce & 42 & 8 & \multirow{4}{*}{$\mathbf{0 , 0 1 1}$} \\
\hline & & $\%$ & $84,0 \%$ & $16,0 \%$ & \\
\hline & \multirow{2}{*}{ severe to critical } & Workforce & 34 & 21 & \\
\hline & & $\%$ & $61,8 \%$ & $38,2 \%$ & \\
\hline
\end{tabular}

Correlations between laboratory and radiological parameters

The correlation between initial lymphocyte count and radiological extension was as follows: 31 patients with severe radiological extension and a mean lymphocyte count of 1201 ( $\mathrm{p}=0.005)$. The correlation between the initial CRP level and radiological extension: 35 patients with severe extension and a mean CRP level of $157 \quad(p=0.001)$. These results are statistically significant with respect to the D-dimer level. 
Table-5: Comparison of biological findings between patients who have mild, severe and critical lung involvement

\begin{tabular}{|c|c|c|c|c|c|}
\hline - & & \multirow{2}{*}{$\begin{array}{l}\mathbf{N} \\
27 \\
\end{array}$} & \multirow{2}{*}{\begin{tabular}{|l|} 
Average \\
1727,19 \\
\end{tabular}} & \multirow{2}{*}{$\begin{array}{l}\begin{array}{l}\text { Standard } \\
\text { deviation }\end{array} \\
628,401\end{array}$} & $p$ \\
\hline \multirow{4}{*}{ Lymphocytes } & mild & & & & \multirow{4}{*}{, 005} \\
\hline & moderate & 31 & 1192,39 & 627,752 & \\
\hline & severe & 31 & 1201,74 & 437,814 & \\
\hline & critical & 12 & 1306,67 & 971,300 & \\
\hline \multirow{4}{*}{ CRP initial } & mild & 28 & 68,923 & 61,3269 & \multirow{4}{*}{,0001 } \\
\hline & moderate & 33 & 125,233 & 88,8701 & \\
\hline & severe & 35 & 157,458 & 111,7739 & \\
\hline & critical & 15 & 222,800 & 161,5011 & \\
\hline \multirow{4}{*}{ D-Dimer } & mild & 11 & 926,182 & 1311,7044 & \multirow{4}{*}{, 058} \\
\hline & moderate & 20 & 1494,000 & 1244,9874 & \\
\hline & severe & 16 & 6119,750 & 12455,8423 & \\
\hline & critical & 8 & 9288,375 & 11799,9263 & \\
\hline
\end{tabular}

\section{Prognosis}

The cohort formed between October and December 2020 included 125 patients with Covid-19, classified into two groups according to severity. The median age of the participating patients was 59.6 years. $73.6 \%$ (92) of the patients left the ICU after completing treatment, $16.8 \%$ (21) were transferred to an intensive care unit, $8.8 \%$ (11) to an inpatient unit, and $0.8 \%(1)$ died. $73.6 \%$ (92) were considered to have a good prognosis (group A) and $26.4 \%$ (33) a poor prognosis (group B).

Table-6: Patient outcome

\begin{tabular}{|l|l|l|}
\hline & Workforce & Percentage \\
\hline Deaths & 1 &, 8 \\
\hline Discharge from the ICU (after completing treatment) & 92 & 73,6 \\
\hline Transfer to inpatient unit & 11 & 8,8 \\
\hline Transfer to intensive care unit & 21 & 16,8 \\
\hline Total & 125 & 100,0 \\
\hline
\end{tabular}

Table-7: Prognostic evolution of the two groups

\begin{tabular}{|l|l|l|}
\hline & Workforce & Percentage \\
\hline Good prognosis (groupe A) & 92 & 73,6 \\
\hline Poor prognosis (groupe B) & 33 & 26,4 \\
\hline Total & 125 & 100,0 \\
\hline
\end{tabular}

The factors that conditioned the prognosis were: âge, lymphocyte count, D-dimer, respiratory rate, saturation and 02 flow.

\section{Correlation between clinical parameters and prognostic evolution}

92 patients with a mean age of 57.9 years +/13.03 had a good prognosis against 33 patients with a mean age of 64.52 years $+/-13.97$ had a poor prognosis with a $(\mathrm{p}=0.016)$.

Table-8: Association of age and prognosis

\begin{tabular}{|l|l|l|l|l|l|}
\hline & Evolution & n & Average & Standard deviation & $\boldsymbol{p}$ \\
\hline \multirow{2}{*}{ AGE } & Good prognosis & 92 & 57,90 & 13,03 & \multirow{2}{*}{$\mathbf{0 , 0 1 6}$} \\
\cline { 2 - 6 } & Poor prognosis & 33 & 64,52 & 13,97 & \\
\hline
\end{tabular}

Respiratory rate: in patients with a respiratory rate $<24$ cycles/min: $85.7 \%$ (48) of them had a good prognosis, $14.3 \%$ (8) a poor prognosis with a $(\mathrm{p}=$ $0.006)$.

Table-9: Association of respiratory rate and prognosis

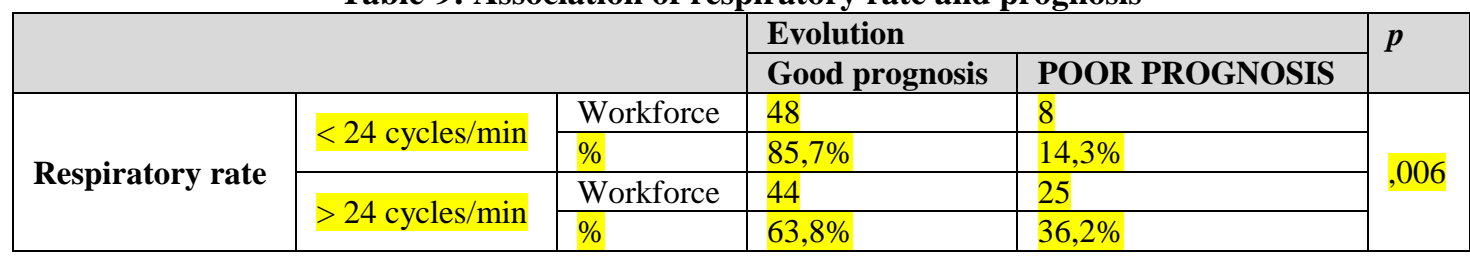


$83.1 \%$ (59) of patients with $>90 \%$ saturation progressed well compared to $61.1 \%$ (33) of patients with $<90 \%$ saturation with a $(\mathrm{p}=0.08)$.
$86.8 \%$ of patients with a good prognosis had an 02 flow $<10 \mathrm{~L} / \mathrm{min}$ while $65.5 \%$ had a poor prognosis with an 02 flow $>10 \mathrm{~L} / \mathrm{min}$ with a $(\mathrm{p}=0.0001)$.

Table-10: Association of saturation and prognostic evolution

\begin{tabular}{|c|c|c|c|c|c|}
\hline \multirow{2}{*}{\multicolumn{3}{|c|}{ 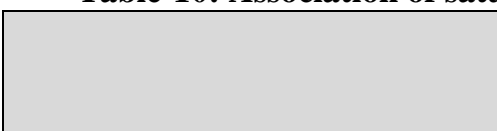 }} & \multicolumn{2}{|l|}{ Evolution } & \multirow[t]{2}{*}{$p$} \\
\hline & & & $\begin{array}{l}\text { Good } \\
\text { prognosis }\end{array}$ & $\begin{array}{l}\text { Poor } \\
\text { prognosis }\end{array}$ & \\
\hline \multirow{4}{*}{ Saturation } & \multirow{2}{*}{$<90$} & Workforce & 33 & 21 & \multirow{4}{*}{,008 } \\
\hline & & $\%$ & $61,1 \%$ & $38,9 \%$ & \\
\hline & \multirow{2}{*}{$>=90$} & Workforce & 59 & 12 & \\
\hline & & $\%$ & $83,1 \%$ & $16,9 \%$ & \\
\hline
\end{tabular}

Table-11: Association between 02 flow rate and prognostic evolution

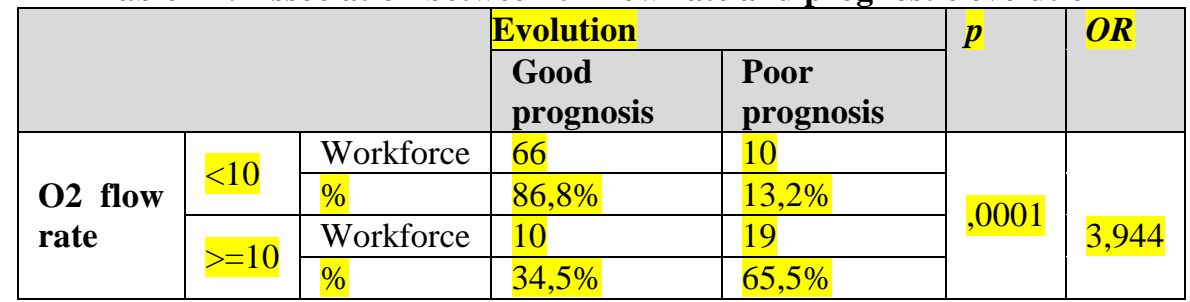

Correlation between biological parameters and prognostic evolution

Lymphocyte count was also a factor that conditioned prognosis: 44 patients with a mean lymphocyte count of 1514 had a good prognosis versus
21 poor prognosis patients with a mean lymphocyte count of 1120 with one $(p=0.037)$. D-dimer assay: 37 patients with a mean d-dimer level of 1265 had a good prognosis versus 18 patients with a mean d-dimer level of 9193 with a $(\mathrm{p}=0.023)$.

Table-12: Association of biological parameters and prognostic evolution

\begin{tabular}{|l|l|l|l|l|l|}
\hline & Evolution & $\mathbf{n}$ & Average & Standard deviation & $\boldsymbol{p}$ \\
\hline \multirow{2}{*}{ Lymphocytes } & Good prognosis & 44 & 1514,23 & 739,15 & \multirow{2}{*}{$\mathbf{0 , 0 3 7}$} \\
\cline { 2 - 6 } & Poor prognosis & 21 & 1120,95 & 597,19 & \multirow{2}{*}{$\mathbf{0 , 0 2 3}$} \\
\hline \multirow{2}{*}{ D-Dimer } & Good prognosis & 37 & 1265,29 & 1093,42 & \multirow{2}{*}{} \\
\cline { 2 - 6 } & Poor prognosis & 18 & 9193,05 & 13435,11 & \\
\end{tabular}

\section{Prognosis evolution of the two groups} $(\mathrm{p}=0.0001)$

Discharge with an oxygen extractor was considered a poor prognostic element in $71 \%$ of patients with one

Table-13: Association between the necessity of 02 extractor and prognostic evolution

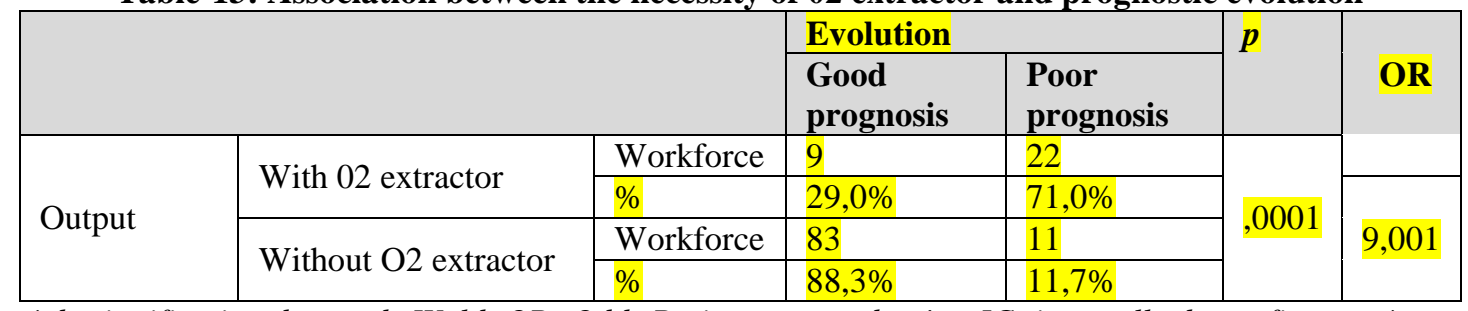

p: degré de signification du test de Wald, OR: Odds Ratio: rapport de côte, IC: intervalle de confiance ; * : p<0,05;

$$
* * *: p<0,00
$$




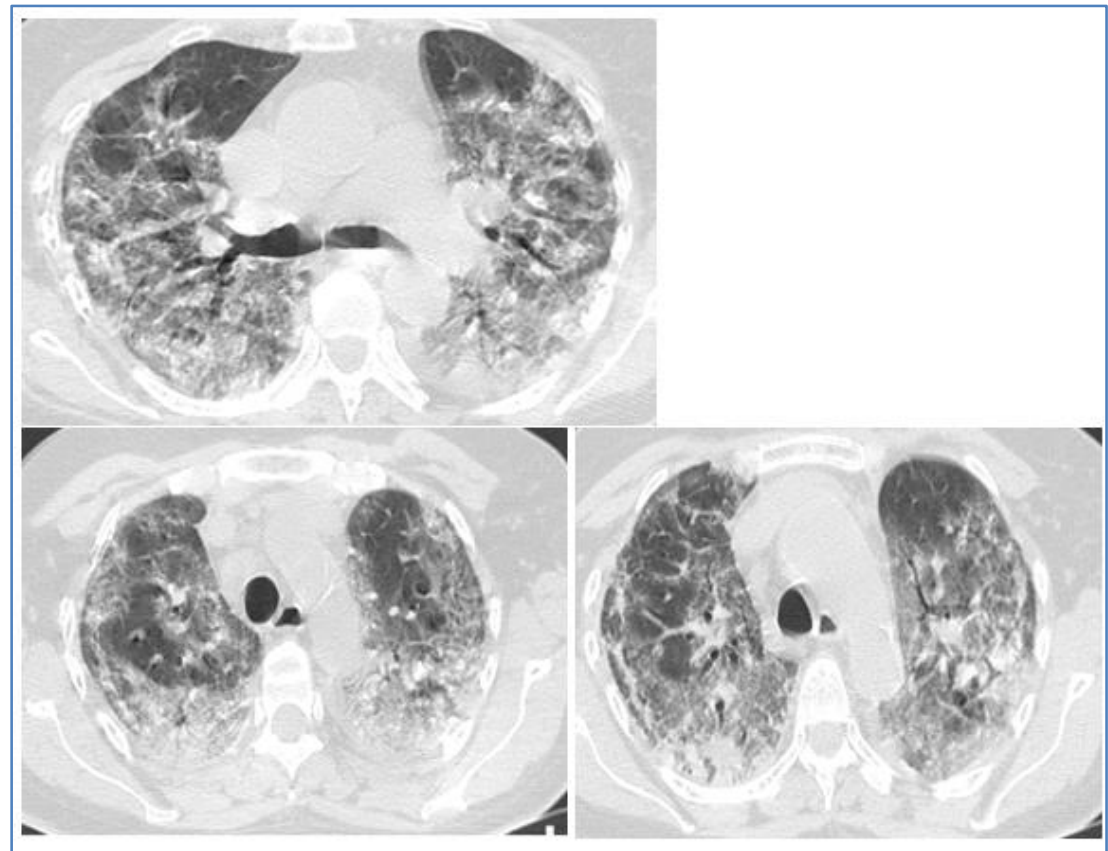

Fig-1: Enhanced thoracic CT: ground glass patch and foci of condensation with an estimated impairment of $>75 \%$, with no evident sign of pulmonary embolism

\section{DISCUSSION}

We report on 125 patients with confirmed SARS-CoV-2 infection, of whom $26,4 \%$ (33) were characterized by severe hypoxemia and admitted to the ICU. This study adds supplementary data about specifics of COVID 19 in Morocco and North Africa. Overall, data from a few thousand analyzed patients show that severe illness can be expected in the elderly, although the younger patients are not protected either.

In most Chinese studies, the age range of severe patients is 52 to 66 years. In the Italian population, the mortality rate increases with age: $12 \%$ in patients over 70 years and $20 \%$ in those over 80 years [1].

In this study, we found that severe patients are aged approximatively $59.6+/-13.53$ years, which is similar to published data from China and Italy.

The study confirms the association between advanced age and increased severity rates in patients with COVID-19, consistent with a previous study. Advanced age is known to be associated with impaired immune function and more underlying comorbidities, which may lead to a poor outcome in these patients.

Severe patients had more diabetes, heart disease, neoplasia, bronchial asthma mellitus and hypertension, as published by Posso $\mathrm{M}$ et al. [2] in a spanish study.

The presence of comorbidities in patients hospitalized with COVID-19 is commun and may negatively affect their prognosis. [3] Previous studies have shown pre-existing diabetes, cardiovascular, or any other chronic diseases can increase the risk of developing severe covid 19 [4] whereas the increase in mortality was mostly associated with cardiovascular diseases.

Cough, and dyspnea were the most common repiratory symptoms in patients with severe COVID-19. A higher body temperature $\left(>38^{\circ} \mathrm{C}\right)$ observed initially, and asthenia, myalgia also appeared to be predictive of worsening disease severity [5].

In a retrospective cohort study among 369 adult patients with COVID-19 admitted to Hospital Cayetano Heredia; a tertiary care hospital in Lima, Peru, Oxygen saturation below $90 \%$ on admission is a strong predictor of in-hospital mortality in patients with COVID-19 [6].

In our study, 71 patients with saturation $>90 \%$ had a mean age of 57.3 years while 54 patients with sao $2<90 \%$ had a mean age of 62.7 with one $(p=0.027)$. As published by Ana B Gómez-Belda, where the study showed that Oxygen saturation $\leq 93 \%$ on room air on admission was a predictor of mortality (odds ratio $11.65,95 \%$ confidence interval $3.26-41.66, P<0.001)$ in patients aged $>70$ years [7].

Regarding radiological examinations, computed tomography (CT) imaging has a high sensitivity for the diagnosis of severe COVID-19 pneumonia. Chest CT revealed a ground glass appearance in $83.2 \%$, alveolar condensations in $40 \%$. Lesions were critical in $12.8 \%$, severe in $30.8 \%$. Radiological extension correlated with saturation: 
$76.1 \%$ of patients with sa02 $>90 \%$ had mild to moderate pulmonary extension with a $(\mathrm{p}=0.0001)$.

The French Society of Radiology in France has proposed a harmonization of the estimation of total lung extension, qualified as mild between 0 and 25\%, moderate between 25 and $50 \%$, severe between 50 and $75 \%$ and critical beyond $75 \%$ (https://ebulletin.radiologie.fr/covid19). However, the association between the extent of radiological lesions thus estimated and the prognosis of the disease has not been established [8].

Some studies have also emphasized the relationship between $\mathrm{CT}$ scan findings and the clinical condition of patients explaining the possible role of lung CT scan in determining the severity and spread of the disease [9].

The aim of our study is to assess the severity of Covid - 19 disease based on its correlation with level of biomarker and radiological severity.

The correlation between initial lymphocyte count, initial crp and radiological extension showed that patients with severe to critical radiological extension had a mean lymphocyte count of 1306 with a significant $\mathrm{p}$ at 0.005 . 50 patients with severe to critical radiological extension had a mean initial crp of 222 . Several inflammatory markers have been identified as predictive of severe disease and/or death.C-reactive protein (CRP) is a well-known marker of inflammation. It is synthesized by hepatocytes under the control of IL 6. Several series have reported increased CRP as a predictive factor for COVID 19.

CRP is a non-specific acute-phase protein induced by IL-6 in liver and sensitive biomarker of inflammation, infection, and tissue damage.18 Studies showed that it increased significantly in severe Covid 19 patients at the initial stage, which is a signal of lung deterioration and disease progression as in Prakhar and al study that demonstrated a significant association between levels of CRP and severity of lung involvement ( $\mathrm{p}=0.0346$, RR of 2.02 , Odds Ratio of 2.37).11 Our study agrees and confirms that CRP levels can be an indicator for severe disease and progressive inflammation $[10,11]$.

The lymphocyte count, although statistically correlated with the occurrence of severity $(p<0.001)$, is not a powerful biomarker in predicting the occurrence of severity $(\mathrm{AUC}=0.222)$. Lymphopenia is the most constant and most observed element in COVID-19 infection, Guan et al. in their large series of 1099 patients, noted it in $83.2 \%$ of them.

It is present in all 3 phases of the infection.The meta-analysis of Huang and Pranata noted that the occurrence of lymphopenia is associated with the occurrence of severity, mortality, ARDS, and admission to intensive care. The lymphocyte count, although statistically correlated with the occurrence of severity $(\mathrm{p}<0.001)$, is not a powerful biomarker in predicting the occurrence of severity $(\mathrm{AUC}=0.222)$ [12].

In our study, CRP and lymphocytes are powerful biomarkers in the prediction of the occurrence of severity in COVID-19 patients. Several studies also demonstrated that a higher level of D-dimer was associated with in-hospital mortality $[13,14]$.

The most suggested mechanism was that the hypercoagulable state, which could be reflected by an elevated D-dimer level, might lead to thrombotic events, resulting in poor outcomes. However, the coagulopathy was thought to result from local and systemic inflammation caused by the coronavirus. Also, D-dimer is known as a biomarker of inflammation [15].

This study was limited by the relatively small number of patients, which may limit statistical power and the inclusion of hospitalized patients exclusively (non-hospitalized patients were not included in the analysis). These limitations may be the cause of statistical bias and therefore of the significant difference identified in demographic and symptomatic characteristics, as well as in laboratory results between groups. Missing data on some variables, such as CT image information and biochemical parameters, may lead to bias in identifying risk factors for mortality in severe patients.

The progression of patients with COVID-19 to worsening appears to be associated with age, cardiovascular and metabolic history, severity of radiological involvement, and observed biological disturbances. This study therefore shows the importance of predictive factors such as âge, lymphocyte and ddimer levels, respiratory rate and oxygen saturation as prognostic factors for the evolution of Covid-19 infection with rapid worsening synonymous with the severity of the evolution. Earlier medical intervention and support on these patients with high risk may reduce the fatality of this disease [16].

\section{CONCLUSION}

The progression of patients with COVID-19 to worsening appears to be associated with age, cardiovascular and metabolic history, severity of radiological involvement, and observed biological disturbances. This study therefore shows the importance of predictive factors such as age, lymphocyte and ddimer levels, respiratory rate and oxygen saturation as prognostic factors for the evolution of Covid-19 infection with rapid worsening synonymous with the severity of the evolution. 


\section{REFERENCE}

1. Zhou, F., Yu, T., Du, R., Fan, G., Liu, Y., Liu, Z., ... \& Cao, B. (2020). Clinical course and risk factors for mortality of adult inpatients with COVID-19 in Wuhan, China: a retrospective cohort study. The lancet, 395(10229), 1054-1062.

2. Posso, M., Comas, M., Román, M., Domingo, L., Louro, J., González, C., ... \& Castells, X. (2020). Comorbidities and mortality in patients with COVID-19 aged 60 years and older in a university hospital in Spain. Archivos de bronconeumologia, 56(11), 756.

3. Guan, W.J., Liang, W.H., Zhao, Y., Liang, H.R., Chen, Z.S., Li, Y.M. (2020). Comorbidity and its impact on 1590 patients with Covid-19 in China: a nationwide analysis. Eur Respir J, 55; 2000547, http://dx.doi.org/10.1183/13993003.00547-2020.

4. Wang, X., Fang, X., Cai, Z., Wu, X., Gao, X., Min, J. (2020). Comorbid chronic diseases and acute organ injuries are strongly correlated with disease severity and mortality among Covid-19 patients: A systemic review and meta-analysis. Research (Wash D C), 2020:2402961, http://dx.doi.org/10.34133/2020/2402961.

5. Zheng, Z., Peng, F., Xu, B., Zhao, J., Liu, H., Peng, J., Li, Q., Jiang, C., Zhou, Y., Liu, S., Ye, C., Zhang, P., Xing, Y., Guo, H., Tang, W. (2020). Risk factors of critical \& mortal COVID-19 cases: A systematic literature review and meta-analysis. $J$ Infect, 81(2); e16-e25. doi: 10.1016/j.jinf.2020.04.021.

6. Mejía, F., Medina, C., Cornejo, E., Morello, E., Vásquez, S., Alave, J., Schwalb, A., Málaga, G. (2020). Oxygen saturation as a predictor of mortality in hospitalized adult patients with COVID-19 in a public hospital in Lima, Peru. PLoS One, 28;15(12):e0244171. doi: 10.1371/journal.pone.0244171. PMID: 33370364; PMCID: PMC7769479.

7. Gómez-Belda, A.B., Fernández-Garcés, M., MateoSanchis, E., Madrazo, M., Carmona, M., PilesRoger, L., Artero, A. (2021). COVID-19 in older adults: What are the differences with younger patients? Geriatr Gerontol Int. Jan;21(1):60-65. doi: 10.1111/ggi.14102.

8. Li, K., Wu, J., Wu, F. (2020). The clinical and chest CT features associa- ted with severe and critical COVID-19 pneumonia. Invest Radiol, 10; http://dx.doi.org/10.1097/RLI.0000000000000672.

9. Aalinezhad, M., Alikhani, F., Akbari, P., Rezaei, M.H., Soleimani, S., Hakamifard, A. (2021). Relationship between CT Severity Score and Capillary Blood Oxygen Saturation in Patients with COVID-19 Infection. Indian J Crit Care Med, Mar; 25(3); 279-283.

10. Soraya, G.V., \& Ulhaq, Z.S. (2020). Crucial laboratory parameters in Covid-19 diagnosis and prognosis. Med Clin (Barc), 155(4); 143-151.

11. Gupta, P., Halani, A., Samuel, T., \& Singh, D. P. (2021). Association of inflammatory biomarkers with radiological severity for COVID-19 patient risk stratification: An Indian perspective. Asian Journal of Medical Sciences, 12(4), 1-7.

12. Huang, I., \& Pranata, R. (2020). Lymphopenia in severe coronavirus disease-2019 (COVID-19): systematic review and meta-analysis. Journal of intensive care, 8, 1-10.

13. Zhang, L., Yan, X., Fan, Q., Liu, H., Liu, X., Liu, Z., \& Zhang, Z. (2020). D-dimer levels on admission to predict in-hospital mortality in patients with Covid-19. Journal of thrombosis and haemostasis, 18(6), 1324-1329.

14. Cummings, M. J., Baldwin, M. R., Abrams, D., Jacobson, S. D., Meyer, B. J., Balough, E. M., ... \& O'Donnell, M. R. (2020). Epidemiology, clinical course, and outcomes of critically ill adults with COVID-19 in New York City: a prospective cohort study. The Lancet, 395(10239), 1763-1770.

15. Agapakis, D. I., Tsantilas, D., Psarris, P., Massa, E. V., Kotsaftis, P., Tziomalos, K., \& Hatzitolios, A. I. (2010). Coagulation and inflammation biomarkers may help predict the severity of community-acquired pneumonia. Respirology, 15(5), 796-803.

16. Bivona, G., Agnello, L., \& Ciaccio, M. (2021). Biomarkers for prognosis and treatment response in COVID-19 patients. Annals of laboratory medicine, 41(6), 540-548. 